\title{
Sacrificial layer-assisted nanoscale transfer printing
}

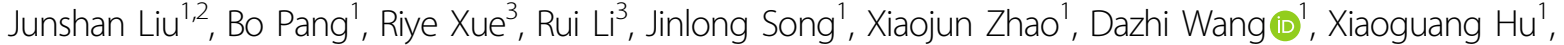 \\ Yao Lu $\mathbb{1}^{4}$ and Liding Wang ${ }^{1,2}$
}

\begin{abstract}
Transfer printing is an emerging assembly technique for flexible and stretchable electronics. Although a variety of transfer printing methods have been developed, transferring patterns with nanometer resolution remains challenging. We report a sacrificial layer-assisted nanoscale transfer printing method. A sacrificial layer is deposited on a donor substrate, and ink is prepared on and transferred with the sacrificial layer. Introducing the sacrificial layer into the transfer printing process eliminates the effect of the contact area on the energy release rate (ERR) and ensures that the ERR for the stamp/ink-sacrificial layer interface is greater than that for the sacrificial layer/donor interface even at a slow peel speed $\left(5 \mathrm{~mm} \mathrm{~s}^{-1}\right)$. Hence, large-area nanoscale patterns can be successfully transferred with a yield of $100 \%$, such as Au nanoline arrays (100 nm thick, $4 \mathrm{~mm}$ long and $47 \mathrm{~nm}$ wide) fabricated by photolithography techniques and PZT nanowires (10 mm long and $63 \mathrm{~nm}$ wide) fabricated by electrohydrodynamic jet printing, using only a blank stamp and without the assistance of any interfacial chemistries. Moreover, the presence of the sacrificial layer also enables the ink to move close to the mechanical neutral plane of the multilayer peel-off sheet, remarkably decreasing the bending stress and obviating cracks or fractures in the ink during transfer printing.
\end{abstract}

\section{Introduction}

Transfer printing is capable of transferring various classes of materials with a wide range of geometries and configurations (referred to as ink) from one substrate (referred to as the donor) to another via a stamp and has been extensively used for flexible and stretchable electronics ${ }^{1}$, such as bendable transistors ${ }^{2}$, stretchable nanophotonic devices ${ }^{3}$, stretchable radio frequency identification tags $^{4}$, and flexible photodetectors ${ }^{5}$. While a variety of transfer printing methods have been developed ${ }^{6-21}$, the resolution of most of these methods, that is, the narrowest continuous line that can be reliably transferred, is at the micrometer scale. There are a few methods that allow the transfer of nanoscale patterns ${ }^{19,20}$. However, such methods require stamps with elaborately designed relief features and specially designed interfacial chemistries at the

\footnotetext{
Correspondence: Junshan Liu (liujs@dlut.edu.cn)

${ }^{1}$ Key Laboratory for Micro/Nano Technology and System of Liaoning Province, Dalian University of Technology, Dalian, Liaoning 116024, China

${ }^{2}$ Key Laboratory for Precision and Non-Traditional Machining Technology of the Ministry of Education, Dalian University of Technology, Dalian, Liaoning 116024, China

Full list of author information is available at the end of the article
}

stamp-ink and ink-substrate interfaces for transferring specific materials; thus far, only metal patterns have been demonstrated ${ }^{19,20,22-25}$. In addition, the thickness of the metal patterns is limited to sizes smaller than $50 \mathrm{~nm}^{22,23}$.

Transfer printing is viewed as the competing fracture of the stamp/ink interface and the ink/substrate interface and is determined by the energy release rates (ERRs) for these two interfaces $\left(G_{\text {stamp/ink }} \text { and } G_{\text {ink/substrate }}\right)^{26}$. Kinetically controlling $G_{\text {stamp/ink }}$ by controlling the peel speed of the stamp from the ink can enable transfer printing to be easily conducted and has been widely exploited ${ }^{27-30}$. However, to our best knowledge, this speed-based transfer printing method has never been used for transferring nanoscale patterns. This is mostly because the ERR is also related to the contact area in addition to the peel speed $^{26,31}$. The contact area at the stamp/ink interface is generally considered to be equal to that at the ink/donor interface because it is believed that the flexibility of the stamp can help obtain a conformal contact ${ }^{27}$, so the effect of the contact area on the ERR is usually neglected. However, we believe that with a decrease of the feature size of the ink, the stamp can only partially contact the

\section{(c) The Author(s) 2020}

\footnotetext{
(cc) Open Access This article is licensed under a Creative Commons Attribution 4.0 International License, which permits use, sharing, adaptation, distribution and reproduction in any medium or format, as long as you give appropriate credit to the original author(s) and the source, provide a link to the Creative Commons license, and indicate if changes were made. The images or other third party material in this article are included in the article's Creative Commons license, unless indicated otherwise in a credit line to the material. If material is not included in the article's Creative Commons license and your intended use is not permitted by statutory regulation or exceeds the permitted use, you will need to obtain permission directly from the copyright holder. To view a copy of this license, visit http://creativecommons.org/licenses/by/4.0/.
} 
ink, and the difference in the contact areas between the stamp/ink interface and the ink/donor interface will increase until $G_{\text {ink/substrate }}$ is much larger than $G_{\text {stamp/ink }}$ and the adhesion between the stamp and the ink is not sufficiently large to pick up the ink, even at an extremely high peel speed. This also explains why Au patterns with feature sizes smaller than $100 \mu \mathrm{m}$ cannot be peeled off from a donor substrate by $3 \mathrm{M}$ Scotch tape in the "drytaping" approach developed by Bao's group ${ }^{6}$.

Here, we present a sacrificial layer-assisted nanoscale transfer printing method. A sacrificial layer was introduced into the transfer printing process to eliminate the effect of the contact area; hence, for the first time, large-area nanoscale patterns, such as 100-nm-thick, 4-mm-long, and 47-nm-wide Au nanoline arrays and 10-mm-long and 63$\mathrm{nm}$-wide lead zirconate titanate (PZT) nanowires, were successfully transferred using only a blank stamp and without the assistance of any interfacial chemistries. Moreover, the presence of the sacrificial layer enabled the ink to move close to the mechanical neutral plane of the multilayer peel-off sheet, remarkably decreasing the bending stress and obviating cracks or fractures in the ink ${ }^{10}$. The successful transfer of a variety of materials with diverse geometries and multilayer structures, such as metal-insulator-metal capacitors, demonstrates some of the capabilities and potential applications.

\section{Results and discussion}

\section{Transfer printing process}

Figure 1a-c schematically illustrates the process of the sacrificial layer-assisted nanoscale transfer printing method. A sacrificial layer is deposited on the surface of a donor substrate, and ink is prepared on the sacrificial layer (Fig. 1a). Ink can be fabricated by a variety of methods, such as photolithography-based methods and electrohydrodynamic jet (e-jet) printing, so the classes and thicknesses of the ink are theoretically not limited. To facilitate separation, the sacrificial layer should have a weak adhesion to the donor substrate. The adhesion of deposited metal films on a polymethylmethacrylate (PMMA) plate is normally weak ${ }^{32}$; therefore, a $\mathrm{Cu}$ layer $(70 \mathrm{~nm})$ is used as the sacrificial layer and sputtered onto the surface of a PMMA donor substrate. Then, a stamp is pressed onto the surfaces of the ink and the sacrificial layer with an average pressure of $6.0 \mathrm{kPa}$. Here, $3 \mathrm{M}$ Scotch tape (cat. 600) is used as the stamp for two reasons. First, $3 \mathrm{M}$ Scotch tape has good adhesion to a variety of materials, which is beneficial for peeling the ink off the donor substrate. Therefore, it has been commonly used as the stamp in many kinds of transfer printing methods ${ }^{6,14,33,34}$. Second, $3 \mathrm{M}$ Scotch tape is commercially available, low-cost, highly flexible, and transparent. Therefore, it is also one of the most popular substrates for flexible devices ${ }^{33,35,36}$. In fact, $3 \mathrm{M}$ Scotch tape with transferred ink can itself be a flexible device in many cases, where the ink does not have to be transferred again from the $3 \mathrm{M}$ Scotch tape to another substrate. The stamp is then peeled off (Fig. 1b). Differing from previously reported methods, the sacrificial layer is directly fabricated on the donor substrate and dominates the contact with the stamp in our method. Therefore, the sacrificial layer actually plays the role of the ink, and the ink is just sandwiched between the sacrificial layer and the stamp. Compared to the ink, the sacrificial layer is a continuous film, and its feature size is considered to be infinite; thus, the effect of the contact area on the ERR can be neglected. Moreover, the sacrificial layer has weak adhesion to the donor substrate. Therefore, the ERR for the stamp/inksacrificial layer interface is ensured to be larger than that for the sacrificial layer/donor interface even at a slow peel speed. In all the following experiments and discussions, unless otherwise specified, the sacrificial layer and the ink above it were picked up and transferred onto the tape at a peel speed of $5 \mathrm{~mm} \mathrm{~s}^{-1}$, which is much slower than the speed used in other studies ${ }^{11,27}$. This slower peel speed makes the operation easier to control and is suitable for wider classes of inks and substrates. The sacrificial layer is removed via chemical etching (Fig. 1c). For example, $\mathrm{Cu}$ can be etched by a mixture of $\mathrm{H}_{4} \mathrm{CeN}_{2} \mathrm{O}_{3}, \mathrm{HClO}_{4}$, and $\mathrm{H}_{2} \mathrm{O}$ (10 g:9 mL:100 mL), and then, the stamp with the transferred ink is rinsed with deionized water and blowdried by nitrogen.

Figure $1 \mathrm{~d}$ shows a 100-nm-thick Au nanoline array transferred onto $3 \mathrm{M}$ Scotch tape $(19 \mathrm{~mm} \times 40 \mathrm{~mm})$. This array contains $99 \mathrm{Au}$ nanolines with a width of $47 \pm 3 \mathrm{~nm}$ and a length of $4 \mathrm{~mm}$ and was originally fabricated on a PMMA donor substrate $(30 \mathrm{~mm} \times 30 \mathrm{~mm} \times 2 \mathrm{~mm})$ with a 70-nm-thick $\mathrm{Cu}$ sacrificial layer based on the edge lithography technique (Supplementary Fig. 1). As expected, Au nanolines were successfully transferred and remained intact with a yield of $100 \%$.

To observe the interface between $\mathrm{Au}$ nanolines and the tape and check whether a conformal contact was achieved, we tilted the specimen at different angles in the scanning electron microscope (SEM); however, the interface was not clearly distinguishable because the $\mathrm{Au}$ lines are too narrow, and the tape has poor electrical conductivity (Supplementary Fig. 2). Therefore, we increased the width of the Au lines. Figure 2a shows three groups of 3- $\mu \mathrm{m}$-wide Au line arrays (1600 Au lines in total) transferred onto tape. The $45^{\circ}$ tilt view SEM images clearly show that the tape was only in partial contact with the $\mathrm{Au}$ lines. Therefore, if there is no $\mathrm{Cu}$ sacrificial layer, then $G_{\text {stamp/ink }}$ would be much smaller than $G_{\text {ink/substrate }}$ since the contact area at the tape- $\mathrm{Au}$ line interface is much smaller than that at the $\mathrm{Au}$ line-PMMA donor substrate interface, and 3- $\mu \mathrm{m}$-wide Au lines would not be picked up by the tape regardless of how fast the peel speed 

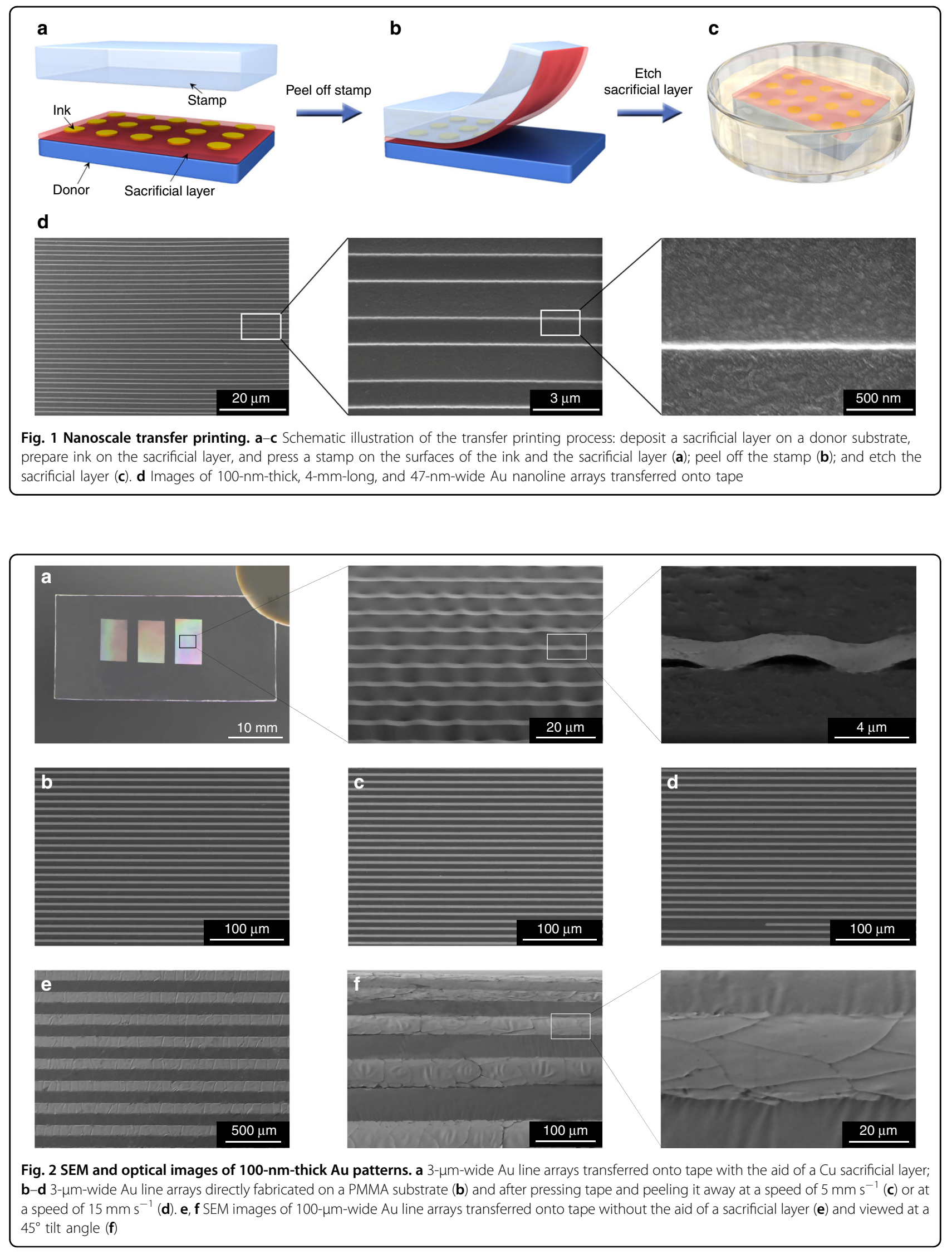


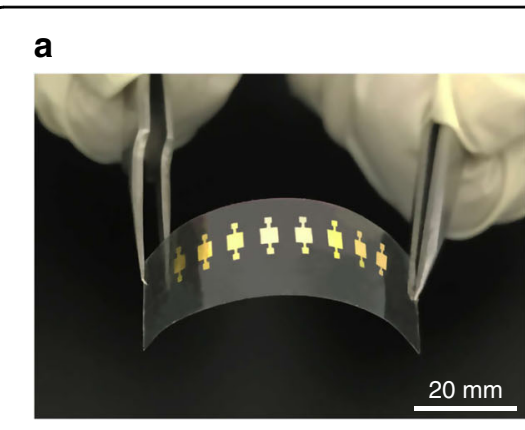

d

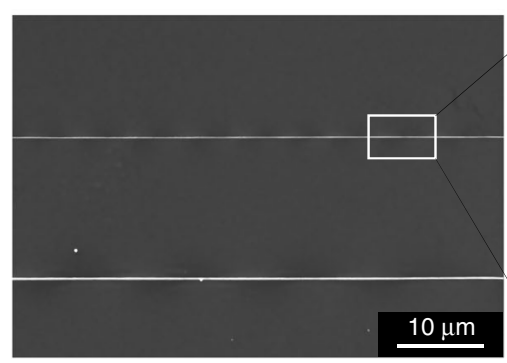

b
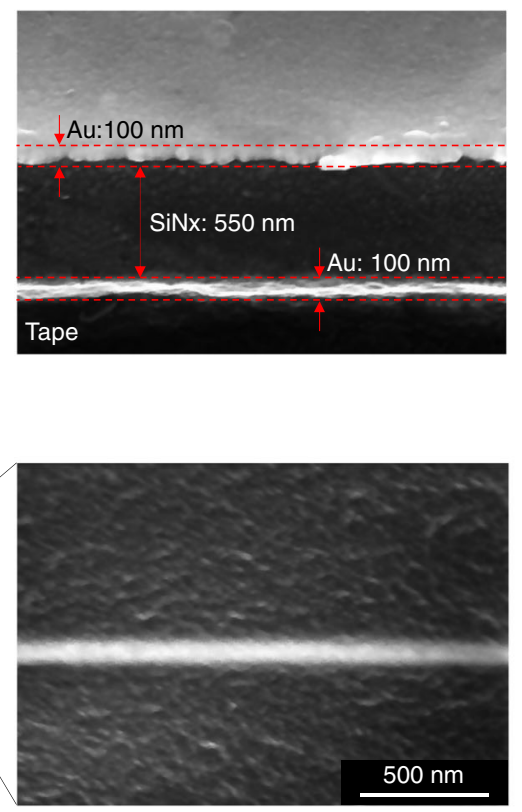

C

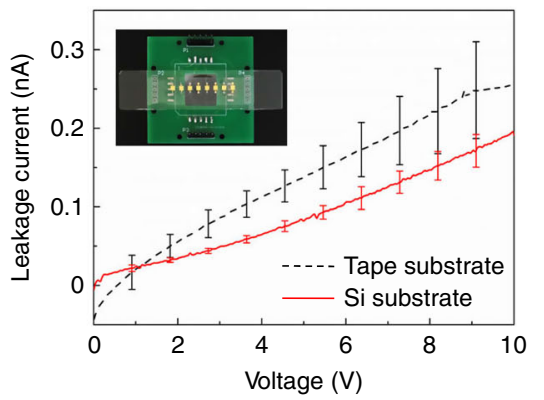

e

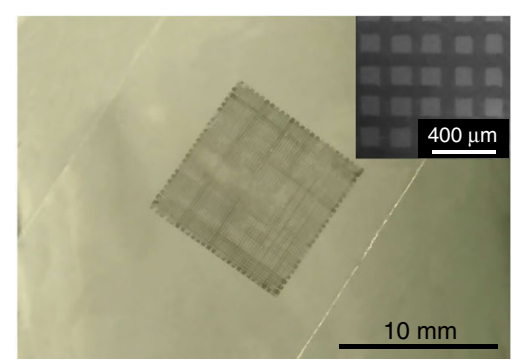

Fig. 3 Micro- and nanostructures transferred from silicon donor substrates. a Eight capacitors transferred onto tape. b SEM image of the cross section of a capacitor viewed at a $45^{\circ}$ tilt angle. c Current-voltage characteristics of capacitors. d PZT nanowires $10 \mathrm{~mm}$ long and $63 \mathrm{~nm}$ wide transferred onto tape. e Graphene patterns transferred onto tape

is. To verify this inference, we fabricated the same 3- $\mu \mathrm{m}$ wide $\mathrm{Au}$ line arrays directly on a PMMA substrate (Fig. $2 b)$. Indeed, the 3- $\mu \mathrm{m}$-wide Au lines could not be picked up at a peel speed of $5 \mathrm{~mm} \mathrm{~s}^{-1}$ (Fig. 2c and Supplementary Video 1), and only small portions of the Au lines were occasionally lifted off even at a fast speed of $15 \mathrm{~mm} \mathrm{~s}^{-1}$ (Fig. 2d and Supplementary Video 2). Nevertheless, when the width of $\mathrm{Au}$ lines directly fabricated on a PMMA substrate was increased to $100 \mu \mathrm{m}$, they could be easily lifted off at a slow peel speed of $5 \mathrm{~mm} \mathrm{~s}^{-1}$ (Fig. 2e and Supplementary Video 3 ), and the $45^{\circ}$ tilt view SEM images show that the tape had an approximately conformal contact with the 100- $\mu$ m-wide Au lines (Fig. 2f). Hence, it can be seen that the contact area does influence the ERR and thus the transfer printing process, and the contact area plays a more important role than the peel speed when the feature size of the ink is smaller than a critical value; the substantial improvement in the resolution of the transfer printing method reported here is indeed attributed to the presence of the sacrificial layer.

\section{Versatility of transfer printing}

The method presented here can essentially transfer almost any class of materials as long as we properly select the sacrificial layer and the donor substrate. On the one hand, the fabrication techniques of the ink should be compatible with the sacrificial layer and the donor substrate.
On the other hand, the sacrificial layer should have a weak adhesion to the donor substrate to facilitate separation. Silicon is compatible with a variety of micro- and nanofabrication techniques, including high-temperature processes that are not compatible with polymers, and it has poor adhesion to weakly reactive metals such as $\mathrm{Cu}, \mathrm{Au}$ or Pt. Therefore, a silicon wafer as the donor substrate and a $\mathrm{Cu}$ or $\mathrm{Au}$ film as the sacrificial layer were selected as another example to examine the versatility and application potential of this method.

Figure 3a shows eight capacitors on tape transferred from a silicon donor substrate with a $\mathrm{Cu}$ sacrificial layer $(70 \mathrm{~nm})$. Each capacitor is composed of a $100-\mathrm{nm}$-thick bottom Au layer, a 550-nm-thick SiNx insulating layer, and a 100-nm-thick top Au layer. The SEM image (Fig. 3b) taken at a $45^{\circ}$ tilt angle shows the cross section of the three-layer structure, which was first cut on the silicon wafer by a diamond pen and then transferred onto the tape. The $\mathrm{SiN}_{\mathrm{x}}$ layer was deposited at $250^{\circ} \mathrm{C}$ by plasmaenhanced chemical vapor deposition (PECVD). This deposition temperature is much higher than the glass transition temperature of PMMA $\left(105^{\circ} \mathrm{C}\right)$, so a PMMA plate could not be used as the donor substrate. All eight capacitors retained their functions after the transfer, and the performance of the capacitors transferred onto the tape was similar to that of the capacitors on the silicon wafer, as shown in Fig. 3c. This experiment also illustrates 

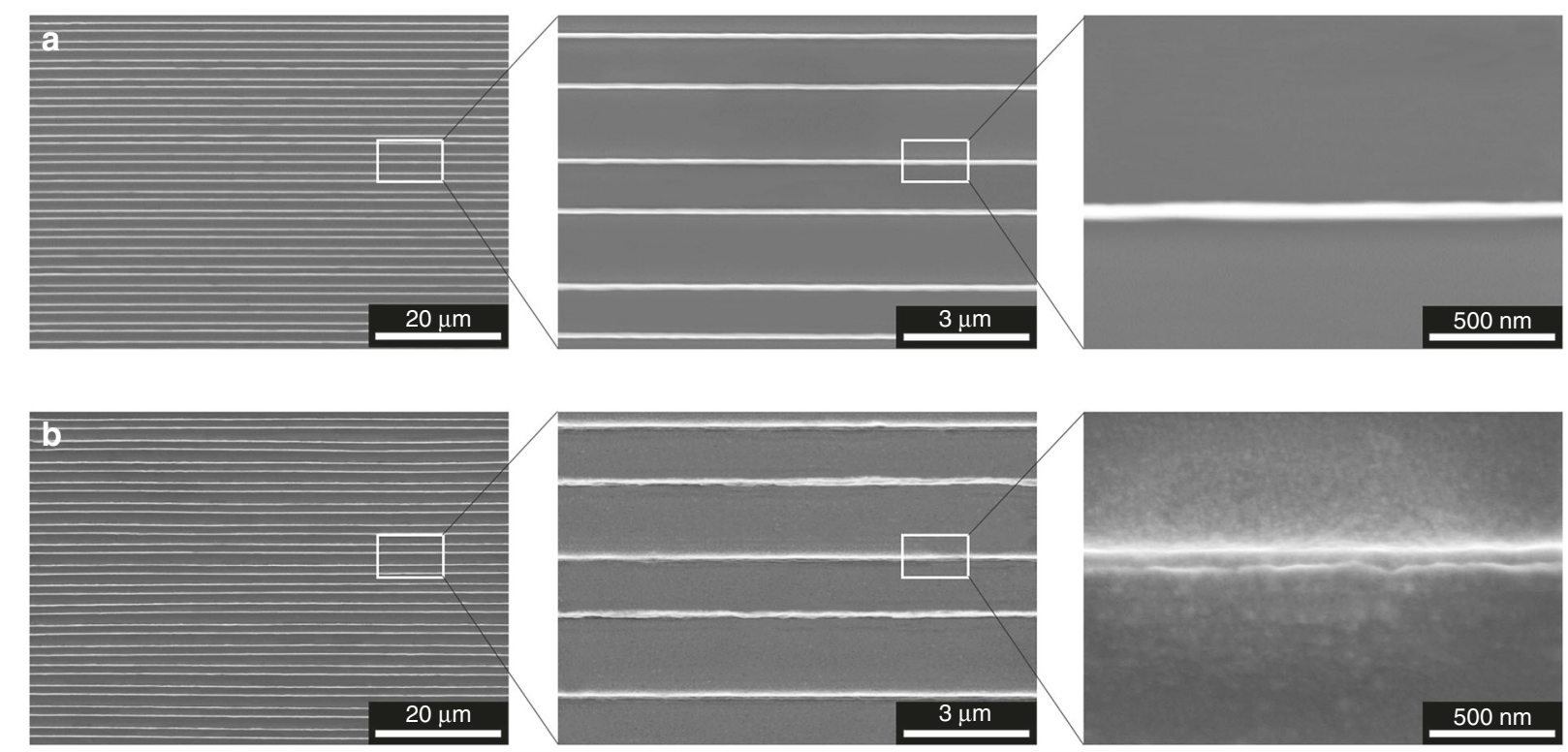

Fig. 4 SEM images of Au nanoline arrays. Au nanoline array fabricated on a PMMA donor substrate (a) and transferred onto a PI receiver substrate by a PDMS stamp (b)

the ability of our method to transfer complex multilayer structures.

E-jet printing is a high-resolution additive manufacturing technique that can directly pattern classes of materials $^{37-39}$. PZT nanowires were first e-jet-printed on a silicon wafer with a $\mathrm{Au}$ sacrificial layer $(70 \mathrm{~nm})$ and sintered at $600{ }^{\circ} \mathrm{C}$ in a muffle furnace. Here, we used a $\mathrm{Au}$ layer to replace the $\mathrm{Cu}$ layer as the sacrificial layer because $\mathrm{Au}$ has higher temperature stability. Then, the $\mathrm{Au}$ layer with PZT nanowires was peeled off from the silicon donor substrate by tape, and it was finally removed via chemical etching by a mixture of $\mathrm{I}_{2}, \mathrm{KI}$, and $\mathrm{H}_{2} \mathrm{O}(1 \mathrm{~g}: 5 \mathrm{~g}: 50 \mathrm{~mL})$. As shown in Fig. 3d, 10-mm-long and $63 \pm 7$-nm-wide PZT nanowires were successfully transferred onto the tape, which further demonstrates the nanoscale resolution of our method. With silicon donor substrates and $\mathrm{a} \mathrm{Au}$ sacrificial layer, e-jet-printed graphene patterns were also successfully transferred onto tape (Fig. 3e).

Furthermore, the lack of requirements for the interfacial chemistries or special relief features over the stamp suggests that the method reported here is suitable for a wide range of other stamps besides $3 \mathrm{M}$ Scotch tape. For example, we used a blank polydimethylsiloxane (PDMS) plate as a stamp and transferred the same structured $\mathrm{Au}$ nanolines as shown in Fig. 1d from a PMMA donor substrate to a polyimide (PI) receiver substrate. Similarly, 99 Au nanolines ( $47 \mathrm{~nm}$ wide and $4 \mathrm{~mm}$ long) were originally fabricated on a PMMA donor substrate $(30 \mathrm{~mm} \times 30 \mathrm{~mm} \times$ $2 \mathrm{~mm}$ ) (Fig. 4a), and a blank PDMS stamp (20 $\mathrm{mm} \times$ $40 \mathrm{~mm} \times 1 \mathrm{~mm}$ ) was pressed on the surfaces of the $\mathrm{Au}$ nanolines and the $\mathrm{Cu}$ sacrificial layer with an average pressure of $6.0 \mathrm{kPa}$. The PDMS stamp was peeled off from the PMMA donor substrate at a speed of $5 \mathrm{~mm} \mathrm{~s}^{-1}$, and the $\mathrm{Cu}$ sacrificial layer was etched away. The PDMS stamp was pressed on the surface of a PI film $(30 \mathrm{~mm} \times 30 \mathrm{~mm} \times$ $75 \mu \mathrm{m})$ (Kapton HN, DuPont, USA) with the same pressure $(6.0 \mathrm{kPa})$. It has been demonstrated that the adhesion between the stamp and the ink is speed sensitive because of the viscoelastic behavior of the stamp, and the adhesion decreases with decreasing separation speed ${ }^{26,27}$. Therefore, to transfer the Au nanowires from the PDMS stamp onto the PI film, the PDMS stamp was peeled off from the PI film at a slow separation speed of $5 \mathrm{~mm} \mathrm{~s}^{-1}$. As shown in Fig. 4b, Au nanolines were successfully transferred onto the PI receiver substrate with a yield of $100 \%$.

\section{Effects of the sacrificial layer on the bending stress}

The ink inevitably endures a bending stress when the stamp is peeled off from the donor substrate. When the stress reaches the failure strength, cracks or even fractures will be induced in the ink ${ }^{10}$. In mechanics, there is a conceptual plane inside a sheet known as the mechanical neutral plane, where the material of the sheet is under no stress during bending. The farther the material is from the mechanical neutral plane, the larger the imposed bending stress is. The presence of the sacrificial layer changed the position of the mechanical neutral plane of the multilayer peel-off sheet and enabled the ink to move close to the mechanical neutral plane (Fig. 5a, b), thus decreasing the bending stress and avoiding cracks or fractures in the ink. 

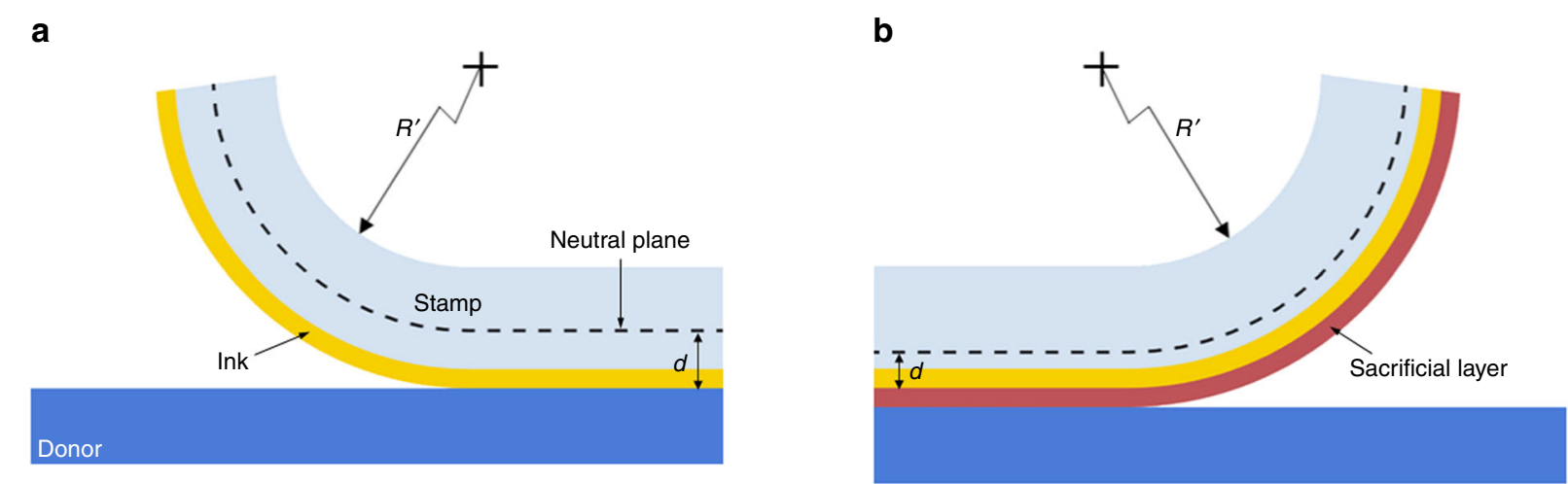

\section{C}

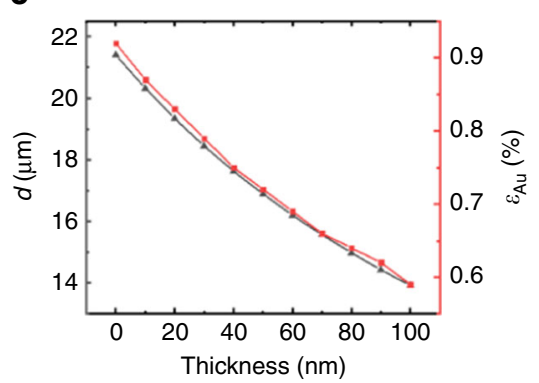

d

e
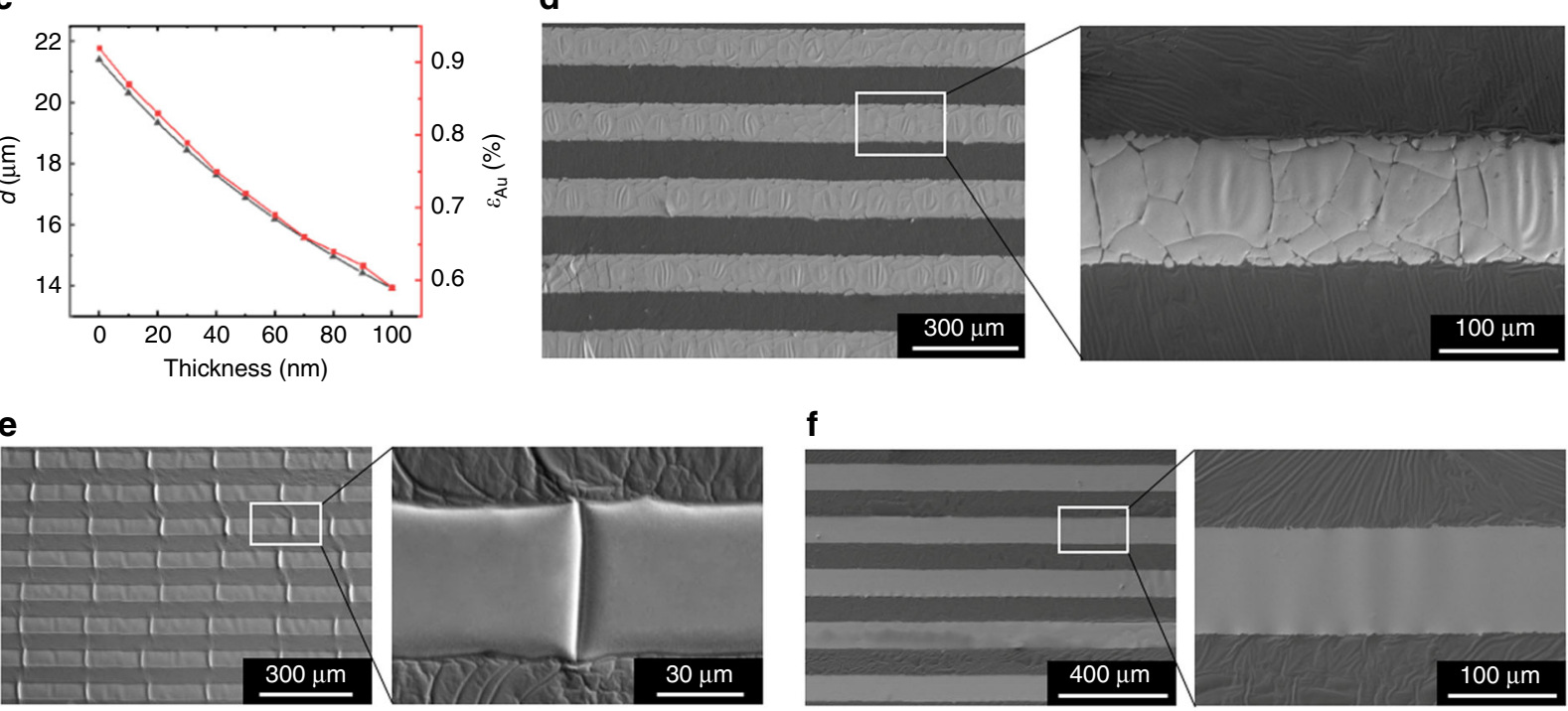

f
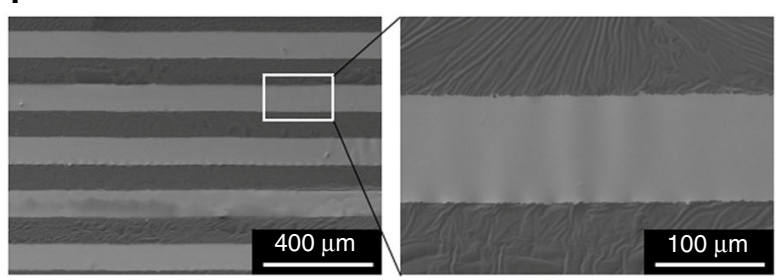

Fig. 5 Effects of the sacrificial layer on the bending stress. $\mathbf{a}$, $\mathbf{b}$ Diagrams of multilayer peel-off sheets without (a) or with (b) a sacrificial layer. The distance between the mechanical neutral plane and ink and the bending curvature radius of the stamp at the advancing edge of the separation region are denoted as $d$ and $R^{\prime}$, respectively. $\mathbf{c}$ Effects of the thickness of the Cu sacrificial layer on the distance $(d)$ and the tensile strain $\left(\varepsilon_{\mathrm{Au}}\right)$. d-f Images of Au line arrays transferred onto Scotch tape without the aid of a Cu sacrificial layer (d) or with the aid of a Cu sacrificial layer with a thickness of $10 \mathrm{~nm}(\mathbf{e})$ or $70 \mathrm{~nm}(\mathbf{f})$

To analyze the effects of the sacrificial layer on the bending stress quantitatively, the multilayer peel-off sheet was studied based on composite beam theory in this work. For the Au-Scotch tape peel-off sheet, where the thickness of the Au layer was constant at $100 \mathrm{~nm}$ and the bending curvature radius of the tape at the advancing edge of the peel region $\left(R^{\prime}\right)$ was constant at $2.3 \mathrm{~mm}$, the detailed theoretical calculation can be seen in Supplementary Note 1. When there was no sacrificial layer, the distance between the bottom of the Au film and the mechanical neutral plane of the peel-off sheet $(d)$ was $24.19 \mu \mathrm{m}$, and the tensile strain in the $\mathrm{Au}$ film $\left(\varepsilon_{\mathrm{Au}}\right)$ caused by the bending force was up to $1.04 \%$. When there was a $\mathrm{Cu}$ sacrificial layer and a 10-nm-thick Cr protection layer, the distance between the bottom of the $\mathrm{Au}$ film and the mechanical neutral plane $(d)$ was

$$
\begin{array}{r}
d=\left(1041357.1-130000 h_{1}^{2}-5390 h_{1}\right) / \\
\left(48630+260000 h_{1}\right)
\end{array}
$$

and the tensile strain $\left(\varepsilon_{\mathrm{Au}}\right)$ in the Au film was

$$
\begin{aligned}
\varepsilon_{\mathrm{Au}}= & \left(1041357.1-130000 h_{1}^{2}-5390 h_{1}\right) / \\
& \left(113633045.9+130000 h_{1}^{2}+613111390 h_{1}\right)
\end{aligned}
$$

where $h_{1}$ denotes the thickness of the $\mathrm{Cu}$ sacrificial layer, with units of microns. According to equations (1) and (2), the effects of the thickness of the $\mathrm{Cu}$ sacrificial layer on the distance between the bottom of the Au film and the 

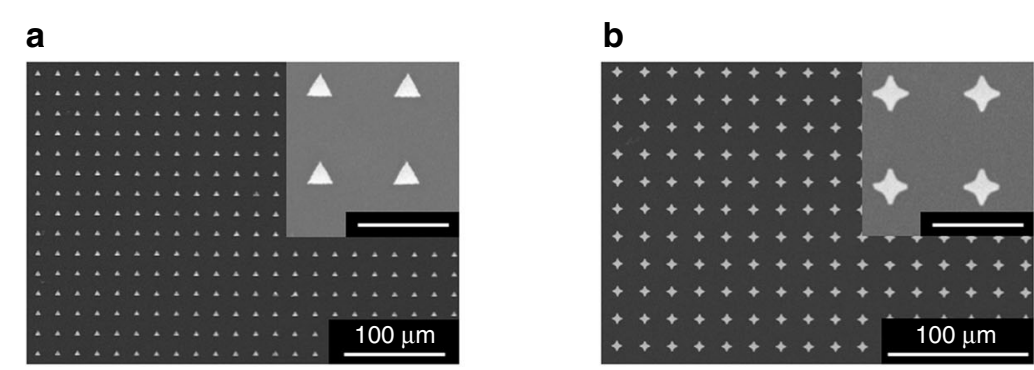

C

d

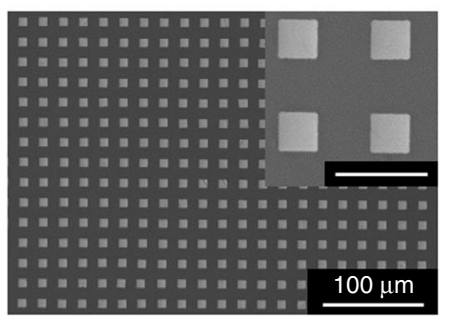

e

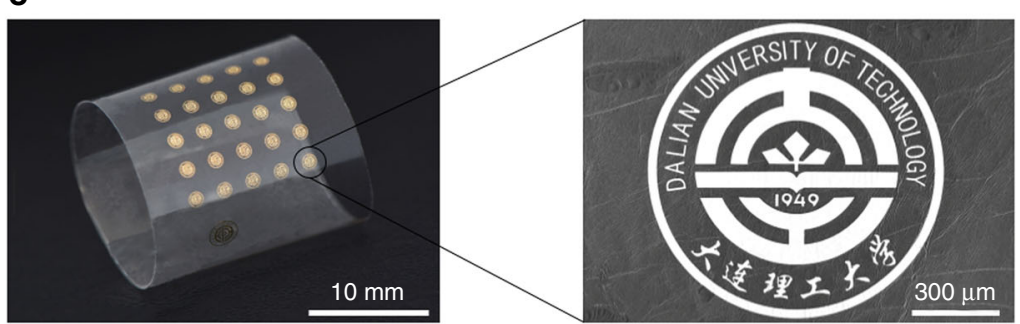

Fig. 6 Images of Au patterns with different shapes and sizes transferred onto tape with the aid of a 70-nm-thick Cu sacrificial layer. The insets of (a-d) are enlarged SEM images (scale: $20 \mu \mathrm{m}$ )

mechanical neutral plane and on the tensile strain in the $\mathrm{Au}$ film are shown in Fig. 5c. It can be seen that with an increase in the thickness of the $\mathrm{Cu}$ sacrificial layer, the distance between the bottom of the $\mathrm{Au}$ film and the mechanical neutral plane decreased, and therefore, the tensile strain in the Au film correspondingly decreased. For example, when the thickness of the $\mathrm{Cu}$ sacrificial layer was $10 \mathrm{~nm}, d$ was $20.32 \mu \mathrm{m}$, and $\varepsilon_{\mathrm{Au}}$ was $0.86 \%$; when this thickness was $100 \mathrm{~nm}, d$ was decreased to $13.92 \mu \mathrm{m}$, and $\varepsilon_{\mathrm{Au}}$ was decreased to $0.59 \%$. The fracture strain of freestanding metal films is typically approximately $1 \%^{40,41}$. Therefore, the fracture of the Au lines can be obviated by optimizing the thickness of the $\mathrm{Cu}$ sacrificial layer. Figure $5 \mathrm{~d}$ shows $\mathrm{Au}$ lines transferred without the assistance of a sacrificial layer, and many tiny cracks or even fractures were observed in the Au lines. When the thickness of the $\mathrm{Cu}$ sacrificial layer was $10 \mathrm{~nm}$, regular cracks were still generated in the transferred Au lines (Fig. 5e). However, when the thickness of the $\mathrm{Cu}$ sacrificial layer was increased to $70 \mathrm{~nm}, \varepsilon_{\mathrm{Au}}$ was $0.66 \%$, the transferred Au lines had a smooth surface, and no cracks were observed (Fig. 5f). With the aid of a 70-nm-thick $\mathrm{Cu}$ sacrificial layer, $\mathrm{Au}$ patterns with a wide range of shapes and sizes were transferred onto Scotch tape, and the magnified SEM images in the insets show that no damage was caused to these Au patterns (Fig. 6a-e).

Nevertheless, we were surprised, though pleased, to find that the ink could sustain a large bending strain once it was transferred onto the stamp. For example, we manually bent $100-\mu \mathrm{m}$-wide $\mathrm{Au}$ lines transferred onto tape around a stainless tube with a $0.5 \mathrm{~mm}$ radius of curvature, and no cracks or fractures were observed in the Au lines, where the tensile strain in the Au film reached $4.53 \%$, which is much larger than the fracture strain of Au films $(\sim 1 \%)^{41}$. Sustaining a large mechanical deformation is of great importance for flexible and stretchable electronics ${ }^{42-44}$. As a demonstration, we fabricated a simple flexible circuit, and this circuit was composed of transferred $\mathrm{Au}$ connectors, three embedded light-emitting diodes (LEDs), and one embedded resistor (Fig. 7a). After 1000 cycles of almost $180^{\circ}$ bending and releasing (Fig. 7b), the Au connectors showed no cracking, and the performance of the circuit showed no obvious changes (Fig. 7c).

\section{Materials and methods \\ Fabrication of microscale Au patterns}

A 70-nm-thick $\mathrm{Cu}$ sacrificial layer was sputtered on the surface of a 2-mm-thick blank PMMA plate purchased from Goodfellow Cambridge Limited (Huntingdon, UK) at a sputtering power of $300 \mathrm{~W}$. To protect the $\mathrm{Cu}$ layer from being etched by the etchant for $\mathrm{Au}$, a 10-nm-thick $\mathrm{Cr}$ protection layer was then sputtered on the $\mathrm{Cu}$ layer at a sputtering power of $150 \mathrm{~W}$. A $100-\mathrm{nm}$-thick Au layer was sputtered on the Cr layer at a sputtering power of $150 \mathrm{~W}$. A positive photoresist (AZ MIR-703) was patterned on the surface of the Au layer. To avoid thermal deformation of the PMMA plate, the photoresist was soft-baked and hardbaked at a low temperature of $60^{\circ} \mathrm{C}$. The exposed gold was etched in a mixture of $\mathrm{I}_{2}, \mathrm{KI}$ and $\mathrm{H}_{2} \mathrm{O}(1 \mathrm{~g}: 5 \mathrm{~g}: 50 \mathrm{~mL})$. The residual photoresist underwent a second exposure to UV light for 3 min without a photomask and was removed by the AZ 300 MIF developer. 

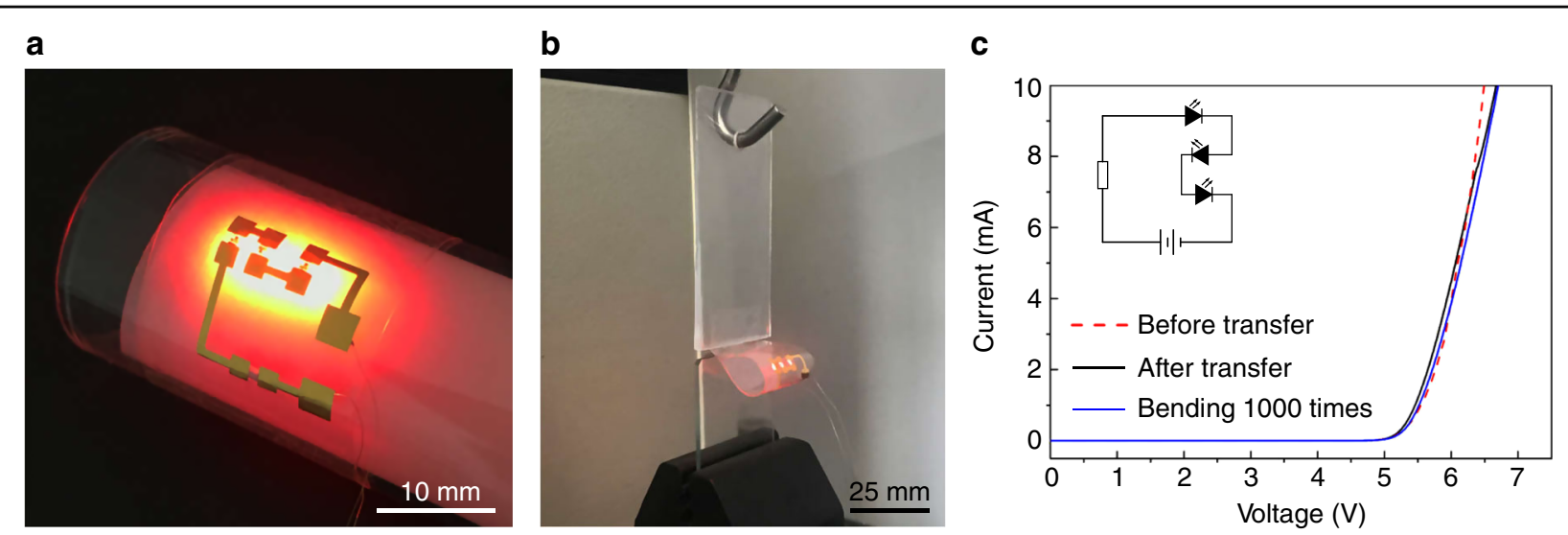

Fig. 7 LED circuits transferred onto tape. a LED circuit bent around a glass tube. b Bending test of LED circuits. c Current-voltage characteristics of LED circuits

\section{Transfer}

3 M Scotch tape (cat. 600) was pressed onto the surfaces of the ink and the sacrificial layer by a finger with an average pressure of $6.0 \mathrm{kPa} \pm 1.3 \mathrm{kPa}$ and then cut to the required dimensions with scissors. The tape was manually peeled off at a peel speed of $5 \mathrm{~mm} \mathrm{~s}^{-1}$. The tape was immersed into the etchant to remove the sacrificial layer. For the $\mathrm{Cu}$ sacrificial layer, the etchant was a mixture of $\mathrm{H}_{4} \mathrm{CeN}_{2} \mathrm{O}_{3}, \mathrm{HClO}_{4}$, and $\mathrm{H}_{2} \mathrm{O}(10 \mathrm{~g}: 9 \mathrm{~mL}: 100 \mathrm{~mL})$, and the $\mathrm{Cr}$ protection layer was simultaneously removed by this etchant. For the Au sacrificial layer, the etchant was a mixture of $\mathrm{I}_{2}, \mathrm{KI}$ and $\mathrm{H}_{2} \mathrm{O}(1 \mathrm{~g}: 5 \mathrm{~g}: 50 \mathrm{~mL})$. The tape was rinsed with deionized water and blow-dried by nitrogen.

\section{Fabrication of capacitors}

A 70-nm-thick $\mathrm{Cu}$ sacrificial layer was sputtered on the surface of a 2-inch silicon wafer. A 10 -nm-thick Cr protection layer was then sputtered on the $\mathrm{Cu}$ layer. A 100$\mathrm{nm}$-thick $\mathrm{Au}$ layer was sputtered on the $\mathrm{Cr}$ layer and then etched to form the top electrode of the capacitor. A 550$\mathrm{nm}$-thick $\mathrm{SiN}_{\mathrm{x}}$ layer was deposited at $250^{\circ} \mathrm{C}$ for $25 \mathrm{~min}$ by PECVD and etched by buffered hydrofluoric acid to form the insulating layer of the capacitor. A positive photoresist (BP212, Beijing Institute of Chemical Reagents, China) was spin-coated and patterned to protect the exposed solder pad of the top electrode from being etched by the etchant for the second Au layer. A second 100-nm-thick $\mathrm{Au}$ layer was sputtered and etched to form the bottom electrode of the capacitor. The residual photoresist was removed by acetone.

\section{E-jet printing}

PZT nanowires were e-jet printed by a coaxial focused electrohydrodynamic jet (CFEJ) printing technique ${ }^{39}$. The homemade equipment for CFEJ printing comprised a computer-controlled three-dimensional movement stage, a coaxial needle unit, two syringe pumps, a high voltage power supply, and a microscopic vision system. The coaxial needle unit was composed of a $130-\mu \mathrm{m}$-diameter inner needle and a 1000- $\mu \mathrm{m}$-diameter outer needle, and the inner needle protruded from the outer needle by $200 \mu \mathrm{m}$. The power supply was connected to the coaxial needle unit to provide an electric field. The two pumps were connected to the inlets of the inner and outer needles to provide the hydrodynamic force. A PZT sol and a high viscosity solution of silicone oil were delivered through the inner and outer needles, respectively, to form a stable coaxial jet. The silicone oil was used mainly because it was immiscible with the PZT sol and highly insulated and could be easily removed. Meanwhile, the silicone oil could protect the PZT sol from environmental disturbances, such as airflow, vibration, and temperature. When an electrical shearing force, the electric fieldinduced high viscous shearing force, and the internal pressure from the silicone oil solution were jointly applied on the PZT sol, the size of the PZT sol jet could be significantly decreased and remained stable at the nanometer scale. The distance between the inner needle and a silicon donor substrate $(10 \mathrm{~mm} \times 10 \mathrm{~mm})$ with a 70 -nm-thick $\mathrm{Au}$ sacrificial layer was $2 \mathrm{~mm}$, a $5.5 \mathrm{kV}$ voltage was applied between them, and the PZT sol encapsulated within the silicone oil was printed on the silicon substrate. The silicon substrate was heated at $200^{\circ} \mathrm{C}$ for $20 \mathrm{~min}$ to evaporate organic solvents and solidify the PZT sol and then immersed in analytical grade isopropanol to remove the silicone oil. Graphene patterns were e-jet printed following a previously reported method ${ }^{38}$. Briefly, ethyl cellulose-dispersed graphene ink was delivered to a 50$\mu \mathrm{m}$-diameter needle at a flow rate of $5 \times 10^{-11} \mathrm{~m}^{3} \mathrm{~s}^{-1}$. The distance between the needle and a silicon substrate with a Au sacrificial layer was $300 \mu \mathrm{m}$. A $1.0 \mathrm{kV}$ voltage was applied, and the graphene ink was printed on the silicon substrate. The silicon substrate was heated at $350^{\circ} \mathrm{C}$ for $30 \mathrm{~min}$ to remove organic solvents. 


\section{Conclusions}

In summary, a simple nanoscale transfer printing method using a weak-adhesion sacrificial layer was developed. Introducing the sacrificial layer into the transfer printing process not only substantially enhances the resolution but also remarkably decreases the bending stress and obviates cracks or fractures in the ink. Its versatility was demonstrated by transferring classes of materials prepared either by top-down or bottom-up techniques. In the near future, the capabilities of the reported method for transferring multiscale structures or repeated transferring will be examined. In addition, applications of the method especially in nanotechnologies where large-area nanopatterning is required, such as surface-enhanced Raman scattering (SERS) and subwavelength optical elements, will be developed.

\section{Acknowledgements}

The authors are grateful for the valuable discussion with Prof. Yonggang Huang at Northwestern University, USA and Prof. Tianhong Cui at the University of Minnesota, USA. This work was supported by the National Natural Science Foundation of China $(51875083,51621064)$. Y. L. acknowledges the support from the QMUL SBCS start-up and the Royal Society Research Grant (RGS\R1\201071).

\section{Author details}

${ }^{1}$ Key Laboratory for Micro/Nano Technology and System of Liaoning Province, Dalian University of Technology, Dalian, Liaoning 116024, China. ${ }^{2}$ Key Laboratory for Precision and Non-Traditional Machining Technology of the Ministry of Education, Dalian University of Technology, Dalian, Liaoning 116024, China. ${ }^{3}$ State Key Laboratory of Structural Analysis for Industrial Equipment, Department of Engineering Mechanics, Dalian University of Technology, Dalian, Liaoning 116024, China. ${ }^{4}$ Department of Chemistry, School of Biological and Chemical Sciences, Queen Mary University of London, London E1 4NS, UK

\section{Author contributions}

J.L. proposed the idea and directed the study. J.L., B.P. X.Z., and X.H. designed and performed the experiments with the help of J.S., D.W., Y.L., and L.W. J.L., B.P., R.X., and R.L. analyzed the data. J.L. and B.P. wrote the paper.

\section{Conflict of interest}

The authors declare that they have no conflict of interest.

Supplementary information accompanies this paper at https://doi.org/ 10.1038/s41378-020-00195-1.

Received: 12 September 2019 Revised: 31 May 2020 Accepted: 9 June 2020 Published online: 21 September 2020

\section{References}

1. Carlson, A. et al. Transfer printing techniques for materials assembly and micro/nanodevice fabrication. Adv. Mater. 24, 5284-5318 (2012).

2. Song, D. et al. High-resolution transfer printing of graphene lines for fully printed, flexible electronics. ACS Nano 11, 7431-7439 (2017).

3. Wang, Q. et al. Tape nanolithography: a rapid and simple method for fabricating flexible, wearable nanophotonic devices. Microsyst. Nanoeng. 4, 31 (2018).

4. Jeong, S., Hjort, K. \& Wu, Z. Tape transfer printing of a liquid metal alloy for stretchable RF electronics. Sensors 14, 16311-16321 (2014).

5. Sim, K. et al. High fidelity tape transfer printing based on chemically induced adhesive strength modulation. Sci. Rep. 5, 16133 (2015).
6. Liu, S. et al. Direct patterning of organic-thin-film-transistor arrays via a "drytaping" approach. Adv. Mater. 21, 1266-1270 (2009).

7. Kim, S. et al. Microstructured elastomeric surfaces with reversible adhesion and examples of their use in deterministic assembly by transfer printing. Proc. Natl Acad. Sci. USA 107, 17095-17100 (2010).

8. Luo, $\mathrm{H}$. et al. Laser-driven programmable non-contact transfer printing of objects onto arbitrary receivers via an active elastomeric microstructured stamp. Natl Sci. Rev. 7, 296-304 (2020).

9. Linghu, C. et al. Rapidly tunable and highly reversible bio-inspired dry adhesion for transfer printing in air and a vacuum. Soft Matter 15, 30-37 (2019).

10. Lee, C. H., Kim, D. R. \& Zheng, X. Fabricating nanowire devices on diverse substrates by simple transfer-printing methods. Proc. Natl Acad. Sci. USA 107, 9950-9955 (2010).

11. Chen, H., Feng, X. \& Chen, Y. Directionally controlled transfer printing using micropatterned stamps. Appl. Phys. Lett. 103, 151607 (2013).

12. Kim, S. J. et al. Ultraclean patterned transfer of single-layer graphene by recyclable pressure sensitive adhesive films. Nano Lett. 15, 3236-3240 (2015).

13. Zhang, Y. et al. A photochemical approach toward high-fidelity programmable transfer printing. Adv. Mater. Technol. 4, 1900163 (2019).

14. Oh, S. et al. Patterned taping: a high-efficiency soft lithographic method for universal thin film patterning. ACS Nano 10, 3478-3485 (2016).

15. Peng, P. et al. One-step selective adhesive transfer printing for scalable fabrication of stretchable electronics. Adv. Mater. Technol. 3, 1700264 (2018).

16. Liu, F. et al. Disassembling 2D van der Waals crystals into macroscopic monolayers and reassembling into artificial lattices. Science 367, 903-906 (2020).

17. Sen, P. et al. Shear-enhanced transfer printing of conducting polymer thin films. ACS Appl. Mater. Interfaces 10, 31560-31567 (2018).

18. Wie, D. S. et al. Wafer-recyclable, environment-friendly transfer printing for large-scale thin-film nanoelectronics. Proc. Natl Acad. Sci. USA 115, E7236-E7244 (2018).

19. Loo, Y. L. et al. Additive, nanoscale patterning of metal films with a stamp and a surface chemistry mediated transfer process: applications in plastic electronics. Appl. Phys. Lett. 81, 562-564 (2002).

20. Smythe, E. J. et al. A technique to transfer metallic nanoscale patterns to small and non-planar surfaces. ACS Nano 3, 59-65 (2009).

21. $\mathrm{Li}, \mathrm{H}$. et al. Elastomers with microislands as strain isolating substrates for stretchable electronics. Adv. Mater. Technol. 4, 1800365 (2019).

22. Loo, Y. L. et al. Interfacial chemistries for nanoscale transfer printing. J. Am. Chem. Soc. 124, 7654-7655 (2002).

23. Loo, Y. L. et al. High-resolution transfer printing on GaAs surfaces using alkane dithiol monolayers. J. Vac. Sci. Technol. B 20, 2853-2856 (2002).

24. Menard, E. et al. Improved surface chemistries, thin film deposition techniques, and stamp designs for nanotransfer printing. Langmuir 20, 6871-6878 (2004).

25. Chanda, D. et al. Large-area flexible 3D optical negative index metamaterial formed by nanotransfer printing. Nat. Nanotechnol. 6, 402-407 (2011).

26. Feng, $X$. et al. Competing fracture in kinetically controlled transfer printing. Langmuir 23, 12555-12560 (2007).

27. Meitl, M. A. et al. Transfer printing by kinetic control of adhesion to an elastomeric stamp. Nat. Mater. 5, 33-38 (2006).

28. $\mathrm{Ko}, \mathrm{H}$. et al. Ultrathin compound semiconductor on insulator layers for highperformance nanoscale transistors. Nature 468, 286-289 (2010).

29. Justice, J. et al. Wafer-scale integration of group III-V lasers on silicon using transfer printing of epitaxial layers. Nat. Photonics 6, 610-614 (2012).

30. Losego, M. D. et al. Effects of chemical bonding on heat transport across interfaces. Nat. Mater. 11, 502-506 (2012).

31. Kim, T. H. et al. Kinetically controlled, adhesiveless transfer printing using microstructured stamps. Appl. Phys. Lett. 94, 113502 (2009).

32. Liu, J. et al. Significant enhancement of the adhesion between metal films and polymer substrates by UV-Ozone surface modification in nanoscale. ACS Appl. Mater. Interfaces 8, 30576-30582 (2016).

33. Fujita, K., Yasuda, T. \& Tsutsui, T. Flexible organic field-effect transistors fabricated by the electrode-peeling transfer with an assist of self-assembled monolayer. Appl. Phys. Lett. 82, 4373-4375 (2003).

34. DeFranco, J. A. et al. Photolithographic patterning of organic electronic materials. Org. Electron. 7, 22-28 (2006).

35. Kim, B. J. et al. A novel method to fabricate parylene-based flexible microfluidic platforms with commercial adhesive tape. J. Micromech. Microeng. 25, 017003 (2015).

36. Guo, R. et al. In-plane micro-supercapacitors for an integrated device on one piece of paper. Adv. Funct. Mater. 27, 1702394 (2017). 
37. Park, J. U. et al. High-resolution electrohydrodynamic jet printing. Nat. Mater. 6 , 782-789 (2007).

38. Wang, D. et al. Electrohydrodynamic jet printing and a preliminary electrochemistry test of graphene micro-scale electrodes. J. Micromech. Microeng. 26, 045010 (2016).

39. Wang, D. et al. Nanoscale coaxial focused electrohydrodynamic jet printing. Nanoscale 10, 9867-9879 (2018).

40. Huang, H. B. \& Spaepen, F. Tensile testing of free-standing $\mathrm{Cu}, \mathrm{Ag}$ and $\mathrm{Al}$ thin films and Ag/Cu multilayers. Acta Mater. 48, 3261-3269 (2000).
41. Lacour, S. P. et al. Stretchable gold conductors on elastomeric substrates. Appl. Phys. Lett. 82, 2404-2406 (2003).

42. Huang, S. et al. Flexible electronics: stretchable electrodes and their future. $A d v$. Funct. Mater. 29, 1805924 (2019).

43. Yang, Y. \& Gao, W. Wearable and flexible electronics for continuous molecular monitoring. Chem. Soc. Rev. 48, 1465-1491 (2019).

44. Linghu, C. et al. Transfer printing techniques for flexible and stretchable inorganic electronics. npj Flex. Electron 2, 26 (2018). 